\title{
Sigmoid carcinoma with sigmoid-rectal intussusception presenting as rectal prolapse and large bowel obstruction in the ED
}

\author{
Pallabi Mazumdar, Pawan Kumar (D), Glory Katiyar, Muniza Mulla and Sanjay Sardessai
}

\begin{abstract}
Background: Intestinal obstruction is a surgical emergency with most cases being small bowel obstruction. Large bowel obstruction is comparatively uncommon and colonic malignancies are a usual cause. Such lesions cause intestinal obstruction by luminal narrowing or rarely serve as lead point of intussusception.

Case presentation: Herein, we describe an unusual case of sigmoid carcinoma causing sigmoid-rectal intussusception, rectal prolapse, and eventually large bowel obstruction.

Conclusion: Pre-operative CT should be done in all adult large bowel obstruction to look for possible site and cause of obstruction and to rule out malignancy as a cause of obstruction. Conversely, every case of rectal prolapse should be diligently evaluated to rule out intussusception, which if present in an adult indicates a high likelihood of underlying malignancy.
\end{abstract}

Keywords: Large bowel obstruction, Intussusception, Rectal prolapse, Rectal carcinoma

\section{Background}

Bowel obstruction is an abdominal emergency with high morbidity and mortality if left undiagnosed. Large bowel obstruction is less frequent than small bowel obstruction. Colorectal carcinoma is the second most common cancer diagnosed in women and third most common cancer in the men [1]. Colonic malignancy is the most common cause of large bowel obstruction, accounting for over $60 \%$ cases [2]. Adult intussusception as a cause of bowel obstruction is rare, accounting for approximately $1-2 \%$ cases. Demonstration of a lead point is seen in more than $80 \%$ cases. More than half of intussusception is associated with malignant lesions including adenocarcinoma, lymphoma and metastasis. The presentation is non-specific, further posing a diagnostic challenge. Some of these patients present with a mass protruding out of the anus, which is often misdiagnosed as

* Correspondence: 0141pawan@gmail.com

Department of Radiology, Goa Medical College and Hospitals, Bambolim Goa 403202, India

\section{Springer Open}

a rectal prolapse, a far more benign cause. We herein present a case of sigmoid carcinoma with sigmoid-rectal intussusception's presenting as rectal prolapse and large bowel obstruction in the emergency department (ED).

\section{Case presentation}

A 52-year-old man came to the ED with the complaint of acute colicky abdominal pain and a mass protruding from the anus, which had progressively increased to its present size in a week. He had a history of constipation for the last 3 months for which he was taking indigenous medicines. There was no history of bleeding per rectum, melena, or other systemic complaints. On physical examination, he had tachycardia with generalized guarding and rigidity of the abdomen. Bowel sounds were sluggish. On local examination, there was large mass measuring approximately $8 \times 5 \times 6 \mathrm{~cm}$ protruding through the anal canal (Fig. 1). Anal sphincter was relaxed, with the prolapsed mass being irreducible. The patients' blood counts 


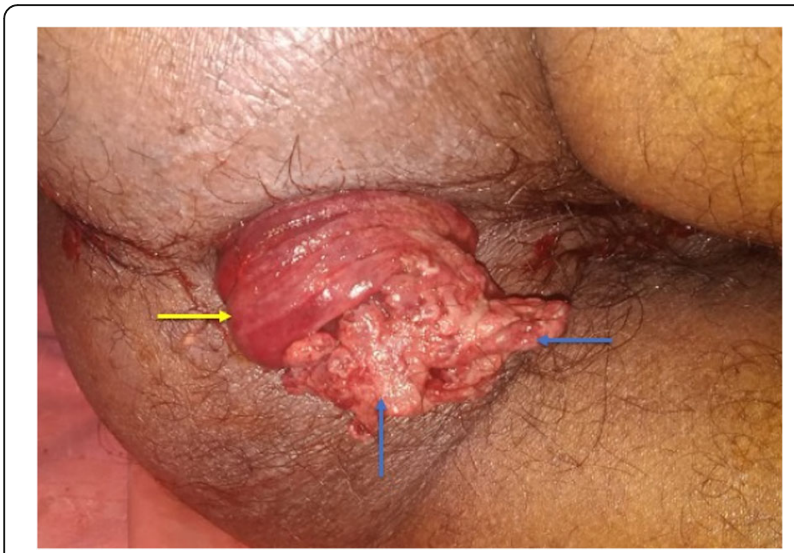

Fig. 1 Prolapsed sigmoid-rectal intussusception (yellow arrows) with mass lesion as lead point (blue arrows)

revealed a normal hemoglobin $(\mathrm{Hb} 11.5 \mathrm{~g} / \mathrm{dl})$, a moderate leukocytosis (WBC of $10,000 / \mu \mathrm{L}$ ) with normal renal function. Plain X-ray abdomen showed dilated left side colon with a gas-less distal bowel. There was no pneumoperitoneum, and no obvious dilated small bowel loops seen (Fig. 2). Further, non-contrast-enhanced computed tomography (NCCT) scan was done which showed a sigmoid-rectal intussusception which was telescoping through the anal canal (Fig. 3). This was associated with proximal bowel dilation suggestive of large bowel obstruction with a distended caecum. There was no significant abdominal lymphadenopathy or metastasis. An emergency laparotomy was performed in view of

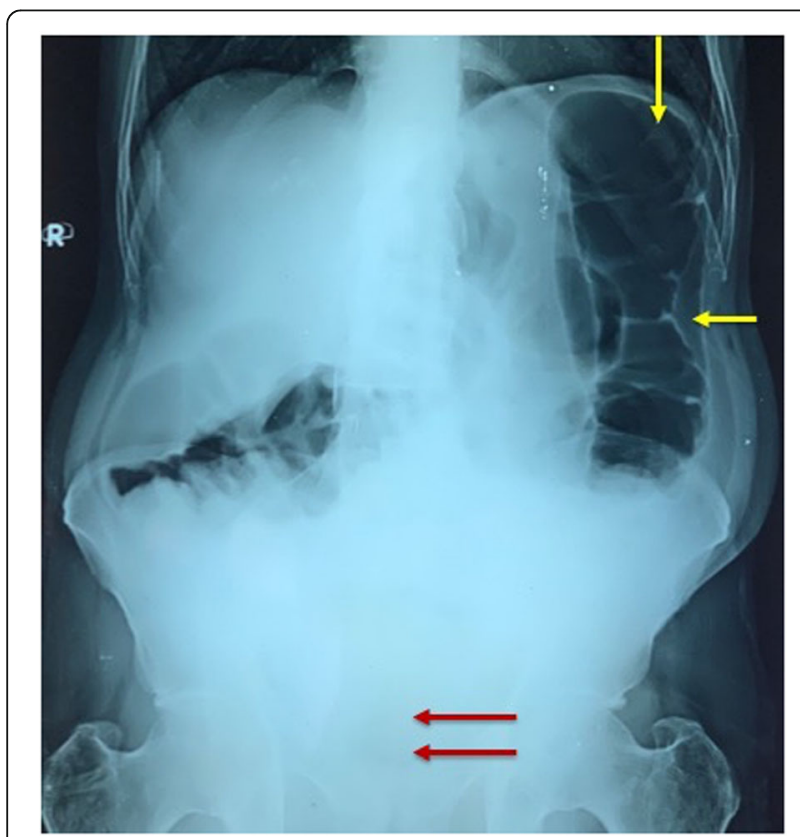

Fig. 2 X-ray abdomen erect AP view image showing decending colon and splenic flexure of colon is grossly dilated (yellow arrows), and there is no rectal gas shadow is seen (red arrows) generalized guarding and rigidity of the abdomen, which revealed an intussusception of the distal sigmoid-rectum with growth, through the anal canal. Hartmann's procedure was performed with resection of the sigmoid growth. The patient was discharged with healthy and functional transverse colostomy. The histopathology was reported as a moderately differentiated adenocarcinoma, infiltrating the submucosa and muscularis propria (Fig. 4). The patient was referred to the oncology department for chemotherapy and had an uneventful course of treatment thereon.

\section{Discussion}

Colorectal carcinoma is one of the commonest malignancies worldwide [1]. While majority of patients present with symptoms like bleeding per-rectum, weight loss, altered bowel habits, $10-29 \%$ of patient with colon cancer can present with the malignant bowel obstruction. Intussusception accounts for $1-2 \%$ of adult large bowel obstruction with primary malignancy being the most common cause of a colo-colic intussusception [3]. Sigmoid carcinoma presenting as large bowel obstruction due to the sigmoid-rectal intussusception with rectal prolapse in the ED is rare. Rectal prolapse refers to protrusion of the part of rectum through the anus. Rarely adenocarcinoma, lymphoma and metastasis, and adenoma of the distal large bowel can act as lead point leading to the intussusception with resulting rectal prolapse $[4,5]$. The association between rectal prolapse and colorectal cancer is rarely reported. Rashid et al. reported a retrospective study of 70 patients with rectal prolapse. In his study, patients with rectal prolapse had a 4.2-fold increased risk of having colorectal cancer (5.7\%), compared with the control group (1.4\%) [6].

Adult intussusception poses a diagnostic dilemma to the clinician due to non- specific clinical presentation and clinical examination findings. Hence, radiology plays a vital role in diagnosis and the management of such cases. Plain abdominal X-rays are usually the first diagnostic tool, demonstrating signs of intestinal obstruction and providing information about probable site of obstruction [7]. Ultrasonography (USG) may demonstrate the typical signs of intussusception [8]. USG is often used in the pediatric population. However, in adults, its utility is limited by air within the bowel lumen and greater depth between the skin and target anatomy.

CT has emerged as a useful imaging modality for diagnosing intussusception in adults and is often performed for primary or secondary assessment of acute and subacute gastrointestinal symptoms of unclear origin. Similar to the USG, CT may also demonstrate target sign, wherein layers of the bowel are duplicated forming concentric rings when imaged at right angles to the lumen. As one image further along the intussusception the mesentery (fat and vessels) 


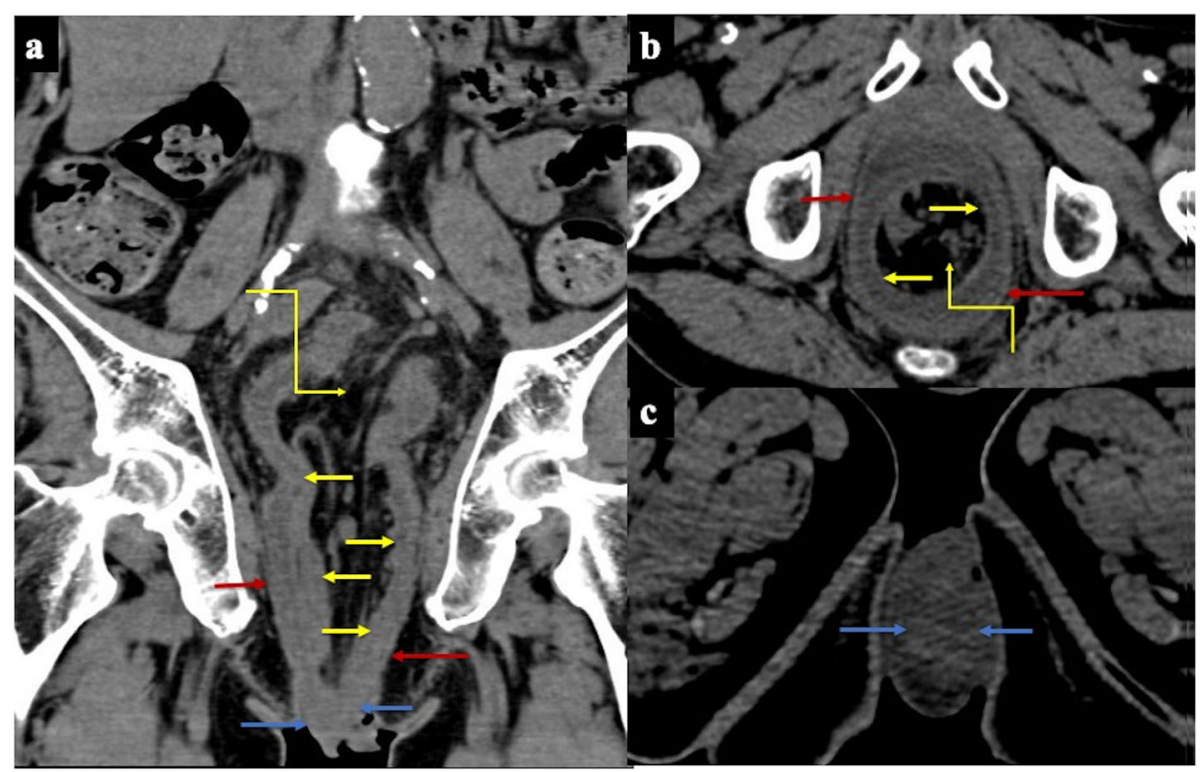

Fig. 3 a-c Coronal and axial non-contrast-enhanced computed tomography (NCCT) images showing intussusception of sigmoid colon (yellow arrows) with its mesentery (curved yellow arrows) into the rectum and anal canal (red arrows) with probable mass lesion as lead point at its tip (blue arrows)

will form a crescent of tissue around the compressed inner most lumen, surrounded by the two layers of the outer enveloping bowel. Even further distally the lead point (if present) will be visualised.

In addition, CT provides greater detail in illustrating local and regional anatomy, which can inform operative planning [9]. In cases of rectal prolapse, special MRI procedures like magnetic resonance (MR) defecography may have several advantages over CT. MR defecography due to its intrinsic dynamic nature, allows accurate assessment of the presence of enterocele, rectocele, and anismus, which are often associated with rectal prolapse

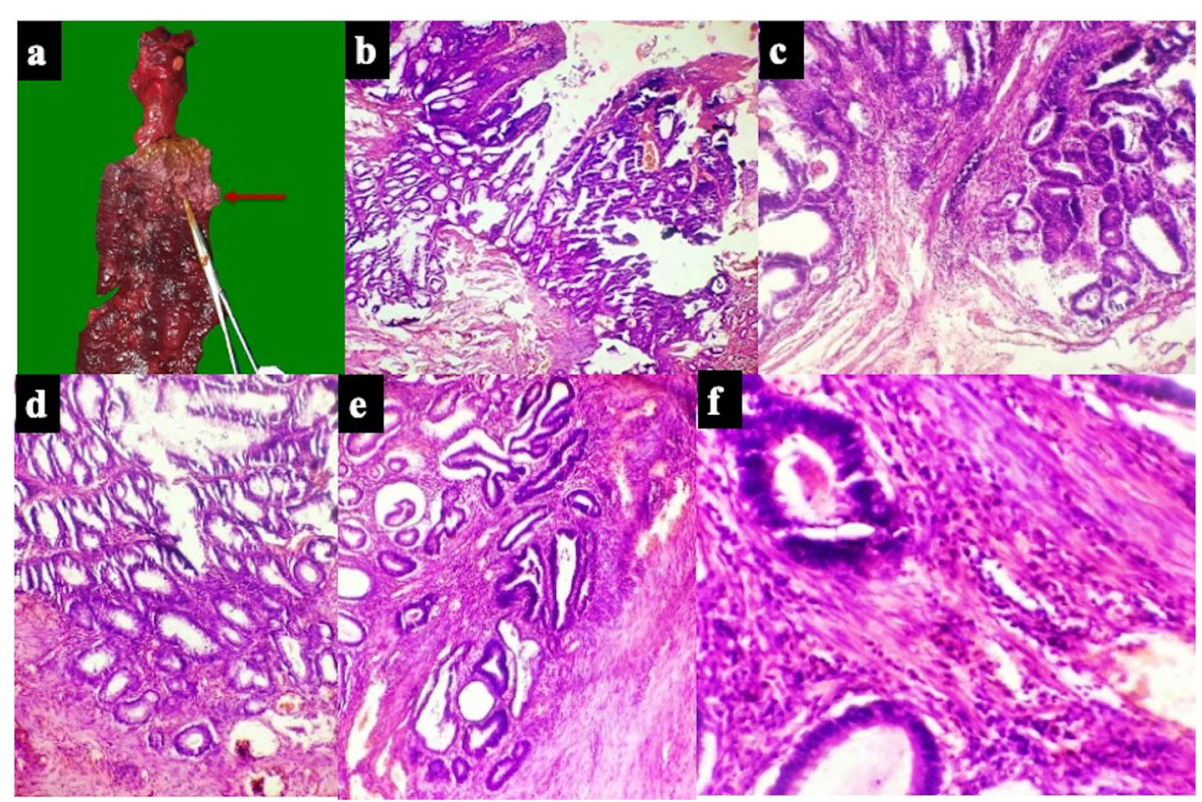

Fig. 4 a Gross image showing a superficial ulcero-proliferative growth in sigmoid (red arrow). b, c Haematoxylin and eosin (H\&E) lower magnification $(\times 40)$ images showing a tumour mainly arranged in glandular pattern. d, e H\&E $(\times 100)$ demonstrating an adenocarcinoma infiltrating in to the underlying lamina propria. $\mathbf{f H \& E}(\times 400)$ shows individual glands having hyperchromatic nuclei with loss of nuclear stratification with intraluminal "dirty" necrosis, along with interspersed area showing mild acute and chronic inflammatory cell infiltrate 
[10]. Endoscopic investigations such as colonoscopy and sigmoidoscopy also play an important role in evaluating the lead point of intussusceptions and obtaining a histology sample but in an acute setting, as in our case, role of MR defecography and sigmoidoscopy is limited. The most common lead point in an adult intussusception is a malignant mass and therefore a routine resection is advocated. This is also because reduction of an intussusception in the presence of a malignant mass may cause intra luminal seeding and venous embolization [11].

\section{Conclusion}

Thus, we can conclude that for early definitive management, a prompt diagnosis of intussusception is imperative and is largely dependent on imaging. CT scan is the primary modality of choice for early diagnosis and surgical planning. Sigmoid-rectal intussusception is an uncommon entity, and it further leading to rectal prolapse is extremely rare occurrence. Hence, it is recommended that all the cases of rectal prolapse should be meticulously evaluated pre-operatively to rule out intussusception and underlying malignancy for better patient management.

\section{Abbreviations}

ED: Emergency department; CT: Computed tomography; WBC: White blood cell; NCCT: Non-contrast-enhanced computed tomography;

USG: Ultrasonography; MRI: Magnetic resonance imaging

\section{Acknowledgements}

None

\section{Authors' contributions}

PM: manuscript preparation. PK: critical revision and editing. GK: literature review. MM: manuscript preparation. SS: manuscript editing. All authors have read and approved the manuscript, and ensure that this is the case.

\section{Funding}

None.

Availability of data and materials

Data, including figures, are available to the reviewers upon request.

\section{Ethics approval and consent to participate}

According to Institute Ethics committee, ethical approval is not required for case reports.

The informed consent form was reviewed and signed by the patient prior to admission.

\section{Consent for publication}

Informed consent was taken from patient.

\section{Competing interests}

All authors declare that they have no competing interests.

Received: 21 September 2020 Accepted: 13 January 2021

Published online: 22 January 2021

\section{References}

1. Bray F, Ferlay J, Soerjomataram I (2018) Global cancer statistics 2018: GLOBOCAN estimates of incidence and mortality worldwide for 36 cancers in 185 countries. CA Cancer J Clin 68(6):394-424

2. Tuca A, Guell E, Martinez-Losada E (2012) Malignant bowel obstruction in advanced cancer patients: epidemiology, management, and factors influencing spontaneous resolution. Cancer Manag Res 4:159-169
3. Jaffe T, Thompson WM (2015) Large-bowel obstruction in the adult: classic radiographic and ct findings, etiology, and mimics. Radiology. 275(3):651-663

4. Yamazaki T, Sakai Y, Sekine $Y$ (1999) Sigmoid colon cancer presenting as complete rectal prolapse: report of a case. Surg Today 29(3):266-267

5. Chen CW, Hsiao CW, Wu CC (2008) Rectal prolapse as initial clinical manifestation of colon cancer. Z Gastroenterol 46(4):348-350

6. Rashid Z, Basson MD (1996) Association of rectal prolapse with colorectal cancer. Surgery. 119(1):51-55

7. Somma F, Faggian A, Serra N et al (2015) Bowel intussusceptions in adults: the role of imaging. Radiol Med 120(1):105-117

8. Cerro P, Magrini L, Porcari P (2000) Sonographic diagnosis of intussusceptions in adults. Abdom Imaging 25(1):45-47

9. Kim YH, Blake MA, Harisinghani MG et al (2006) Adult intestinal intussusception: CT appearances and identification of a causative lead point. Radiographics. 26(3):733-744

10. Colaiacomo MC, Masselli G, Polettini E et al (2009) Dynamic MR imaging of the pelvic floor: a pictorial review. Radiographics. 29(3):e35

11. Filiberto AC, Loftus TJ, Tan SA (2019) Sigmoido-rectal intussusception. SAGE Open Med Case Rep 7:2050313X19856242

\section{Publisher's Note}

Springer Nature remains neutral with regard to jurisdictional claims in published maps and institutional affiliations.

\section{Submit your manuscript to a SpringerOpen ${ }^{\circ}$ journal and benefit from:}

- Convenient online submission

- Rigorous peer review

- Open access: articles freely available online

- High visibility within the field

- Retaining the copyright to your article

Submit your next manuscript at $>$ springeropen.com 